\title{
Redicinal Plant Species Used in the Management of Hernia by Traditional Medicine Practitioners in Central Uganda
}

\author{
Moses Sserwano Kibuuka and Godwin Anywar
}

\section{Research}

\begin{abstract}
Hernia is a common neglected disease that occurs when a portion of tissue or a body organ bulges through a weakened muscle area. This study was carried out to document traditional concepts of hernia and the medicinal plant species used in its treatment among Prometra Traditional Medical Practitioners (TMP) in Mpigi District, central Uganda. Such specialized knowledge is held by a few members of the community, particularly TMPs. Since it is not documented, it is at risk of being forever lost, despite its potential to contribute towards improved healthcare delivery. Data were collected through snowballing and focus group discussions using semi-structured interviews. Thirty TMPs were interviewed. Fifty-one plant species belonging to 28 families were documented. Trees $(49 \%)$ were the most commonly used life form. Roots (32\%) and bark $(30 \%)$ were the most commonly used plant parts. All the medicines were freshly prepared and administered orally. Most medicines (96\%) were prepared as decoctions. Hernia was diagnosed through physical examination, and treatment varied between 1-4 months. In conclusion, the TMPs believe a monotonous starchy diet can cause hernia by straining the intestinal walls. Hernia is treated using various locally available medicinal plant species.
\end{abstract}

\section{Introduction}

Hernia occurs when a portion of tissue or organ in the body bulges into a defect or penetrates through a weakened muscle area (Zollinger 2003). Most surface hernias develop in the abdominal wall (Ross 2003, Zollinger 2003). The main anatomical types of hernias are inguinal and femoral (Dabbas et al. 2011, Zollinger 2003). Inguinal hernias are the most common and most clinically significant, especially among men, where they substantially increase with aging (Ruhl \& Everhart 2007). The prevalence of non-surgically treated inguinal hernia alone was reported to be between 5 and $10 \%$ among men in international and US surveys (Beddy et al. 2006, Everhart 1994). According to Kingsworth and LeBlanc (2003), it was estimated that more than 20 million hernias were repaired annually around the world. This made hernia repair one of the most commonly performed general surgical procedures worldwide (Tekatli et al. 2012)

Prevalence of hernia in Africa is difficult to determine because of insufficient medical records in most parts of the continent. Even in the west, large-scaled epidemiologic data are sparse (Burcharth et al. 2013). However in eastern Uganda alone, the prevalence of groin hernias in adult males was reported to be almost $10 \%$ (Odula 2011). Untreated, hernias may develop potentially fatal complications when they become irreducible or strangulated (Ruhl \& Everhart 2007, Zollinger 2003). A study by Burcharth et al. (2013) in Denmark showed that out of 5,639,885 persons, $88.6 \%$ males and $11.4 \%$ females had at least one groin hernia repair during the five-year study period.

\section{Correspondence \\ Moses Sserwano Kibuuka, Godwin Anywar, Depart- ment of Biological Sciences, Makerere University, P.O. Box 7062 Kampala, UGANDA. godwinanywar@gmail.com ganywar@.cns.mak.ac.ug}

Ethnobotany Research \& Applications 14:289-298 (2015) 
Inguinal hernias comprised $97 \%$ of groin hernia repairs. In comparison, a study carried out by Odula and Kakande (2004) at Mulago National Referral Hospital in Uganda indicated a $30.8 \%$ rate of post-operative complications arising from wound infections, hemorrhages, and chest infections associated with hernia repair. Tharappel et al. (2014) also reported high rate of recurrence of hernias as a persisting problem despite advancements in prosthetic materials technology and surgical procedures in the western world. Hernia surgery may impair testicular function and may ultimately affect male fertility (Beddy et al. 2006, Tekatli et al. 2012).

Although hernias occur universally and constitute a significant disease burden even in Africa (Everhart 1994, Odula \& Kakande 2004), they are often neglected. This has been worsened by (1) the fact that indigenous knowledge and practices on hernia treatment have received little or no attention, (2) inaccessibility of modern health facilities especially in rural areas in developing countries, and (3) the high cost of surgery (Odula 2011, Tabuti et al. 2003). In order for people to survive under such unfavorable conditions, they rely on herbal medicine for their health care (Tabuti et al. 2003). However, such knowledge is at risk of being lost because of rapid modernization, acculturation, and its oral method of transmission (Njoroge \& Kibunga 2007, Tabuti et al. 2010).

The most important step in the conservation of traditional medicinal knowledge is the inventory of plants with their therapeutic value and knowledge related to use in systematic ways (Tabuti et al. 2003). The use of medicinal plants in the treatment of hernia has been documented in different parts of Africa including Tanzania (Amri \& Kisangau 2012) and Uganda by Tabuti et al. (2003) and Gradé et al. (2009). This study is premised on the hypothesis that Traditional Medical Practitioners (TMPs) have traditional concepts of diseases they treat in a community, even diseases like hernia, which are treated surgically in western medicine with several hypotheses regarding their etiology being proposed (Burcharth et al. 2013). In traditional healing systems, alternative traditional treatment options using locally available resources-especially medicinal plants-are available.

The purpose of this study was to investigate the traditional concept of hernia and to document the medicinal plants species and practices used in its treatment by TMPs from Prometra Uganda (Promocion de la medicina tradicional amazonica). A total of 30 TMPs were interviewed during the study. There were more females $(70 \%)$ than males $(30 \%)$ interviewed. All the respondents interviewed were actively using medicinal plants to manage hernia. The traditional healers described hernia as a protrusion of the intestine around the abdominal cavity. The following research questions guided the execution of this study: (1) Do TMPs treat hernia in their communities? (2) What is the traditional concept or understanding of hernia? (3)
What plant species are used in treating hernia and how are they prepared and administered? (4) What practices are associated with the treatment of hernias by TMPs?

\section{Materials and Methods}

\section{Study area}

The study was carried out in Buwama Subcounty, Mpigi District, central Uganda, among Prometra TMPs. The study area is located $64 \mathrm{~km}$ along Masaka Road in central Uganda between $0^{\circ} 32^{\prime} 39^{\prime \prime} \mathrm{N}$ and $32^{\circ} 5^{\prime} 47^{\prime \prime} \mathrm{E}$ at an altitude 1182-1341 m above sea level (UDIH 2012). Mpigi District has a tropical equatorial climate with a mean annual rainfall of $1513 \mathrm{~mm}$. (UDIH 2012). It covers an area of 1526 $\mathrm{km}^{2}$ with a total population of 251,512 according to the provisional results of the National Population and Housing Census (NPHC 2014). The main ethnic group is Baganda (UDIH 2012).

\section{Study design}

An ethnobotanical survey was conducted among TMPs. The study area was purposely chosen because PrometraUganda hosts several herbalists from Uganda once every week. This made it easy for the researchers to target several TMPs in the same area for the survey. The purpose of the study was explained to the healers before the study was conducted. Prior informed consent was obtained from all the healers before the study was conducted. Only those TMPs who consented took part in the study.

\section{Data collection}

The study was conducted between March and August 2014. Data were collected using questionnaires and semi-structured interviews. The TMPs $(\mathrm{N}=30)$ were interviewed after being selected using the snowball technique described in Heckathorn (2011). They were asked what caused hernia, how they diagnosed it, what medicinal plants they used to treat it, how they prepared them, and where they harvested them from. The interviews were conducted in Luganda, the local language spoken in the area.

\section{Plant collection}

Specimens of the mentioned plant species with representative morphological features were collected from the surrounding forested areas under the guidance of the TMPs. The specimens were collected in triplicate following standard collection procedures described in Martin (1995) and deposited at the Makerere University herbarium for taxonomic identification. The scientific names of the plant species were verified using the Kew database at 


\section{Kibuuka \& Anywar - Medicinal Plant Species Used in the Management of Hernia by Traditional Medicine Practitioners in Central Uganda}

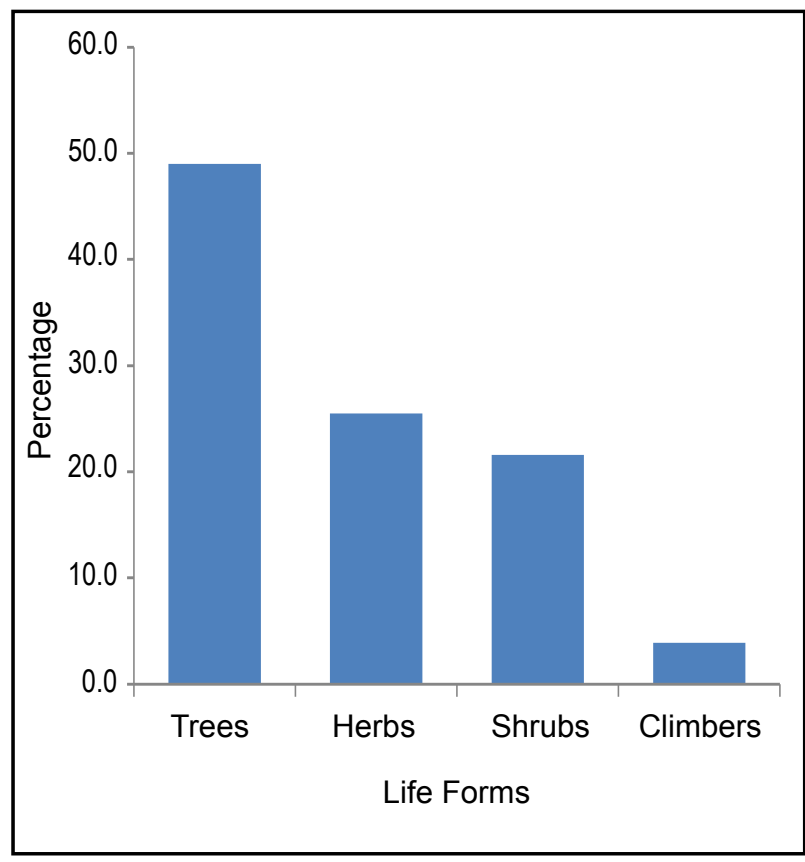

Figure 1. Life forms of medicinal plants used to manage hernia by Traditional Medical Practitioners in Mpigi District, Uganda.

http://www.theplantlist.org (Version 1.1) accessed on $11 \mathrm{Au}$ gust2014, andtheplantfamilieswereinconformitytoAPGIIIdatabase at http://www.mobot.org/MOBOT/research/APweb/.

\section{Results}

A total of 51 plant species belonging to 28 families were recorded as being used to manage hernia (Table 1). The TMPs did not report any specific cause of hernia, but some attributed it to weaknesses in the intestinal wall which could be caused by a monotonous starchy diet consisting mainly of cassava (Manihot esculeta Crantz) and sweet potato (Ipomoea batatas (L.) Lam.). Hernias were diagnosed through physical examination of the patients who presented with abdominal and groin complaints, such as pain and swellings.

The TMPs reported that patients who had not undergone surgery recovered faster than those who had undergone surgery. They also reported successful healing on completion of the dose by the patient. On average, the hernia treatment lasted three months. Most of medicinal plant species used in the management of hernia belonged to Fabaceae (7) and Asteraceae (6) (Table 1). Euphorbiaceae and Lamiaceae both had 4 plant species, while Poaceae had 3 plant species. Anacardiaceae, Rosaceae, Myrtaceae, and Rutaceae had two plant species each. The rest of the 19 families had one plant species each.

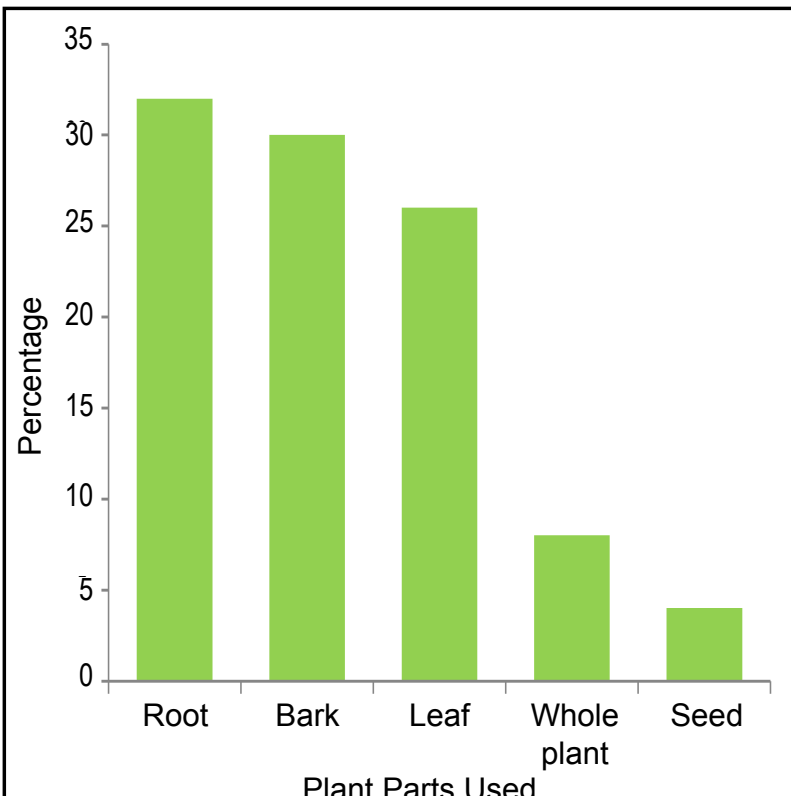

Plant Parts Used

Figure 2. Plant parts used in the preparation of herbal remedies for hernia by Traditional Medical Practitioners in Mpigi District, Uganda.

\section{Plant life forms used}

The most frequently used plants were trees (49\%) followed by herbs $(25.5 \%)$ and shrubs $(21.6 \%)$. Climbers were the fewest at 3.9\% (Figure 1). The most commonly used plant species to manage hernia were Cyphostemma adenocaule (Steud. ex A.Rich.) Desc. ex Wild \& R.B.Drumm. (37\%), Shirakiopsis elliptica (Hochst.) Esser (27\%) Citrullus colocynthis (L.) Schrad. (23\%) and Ocimum americanum L. (23\%), as indicated by the relative frequencies of mention (Table 1).

\section{Conservation status of the medicinal plants}

The majority $(63 \%)$ of the medicinal plants grew in the wild, whereas $23 \%$ were both cultivated and found in the wild. Only $14 \%$ were cultivated in home gardens (Table 1 ), especially the herbaceous fast-growing plant species such as Phaseolus lunatus L. and O. americanum.

\section{Plant parts used, methods of preparation, and application}

Table 1 shows the different plant parts used in the preparation of herbal remedies for the management of hernia. Roots were the most frequently used plant parts $(32 \%)$, followed by bark $(30 \%)$, leaves $(26 \%)$, whole plant $(8 \%)$, and seeds (4\%) (Figure 2). Decoctions were the most fre- 


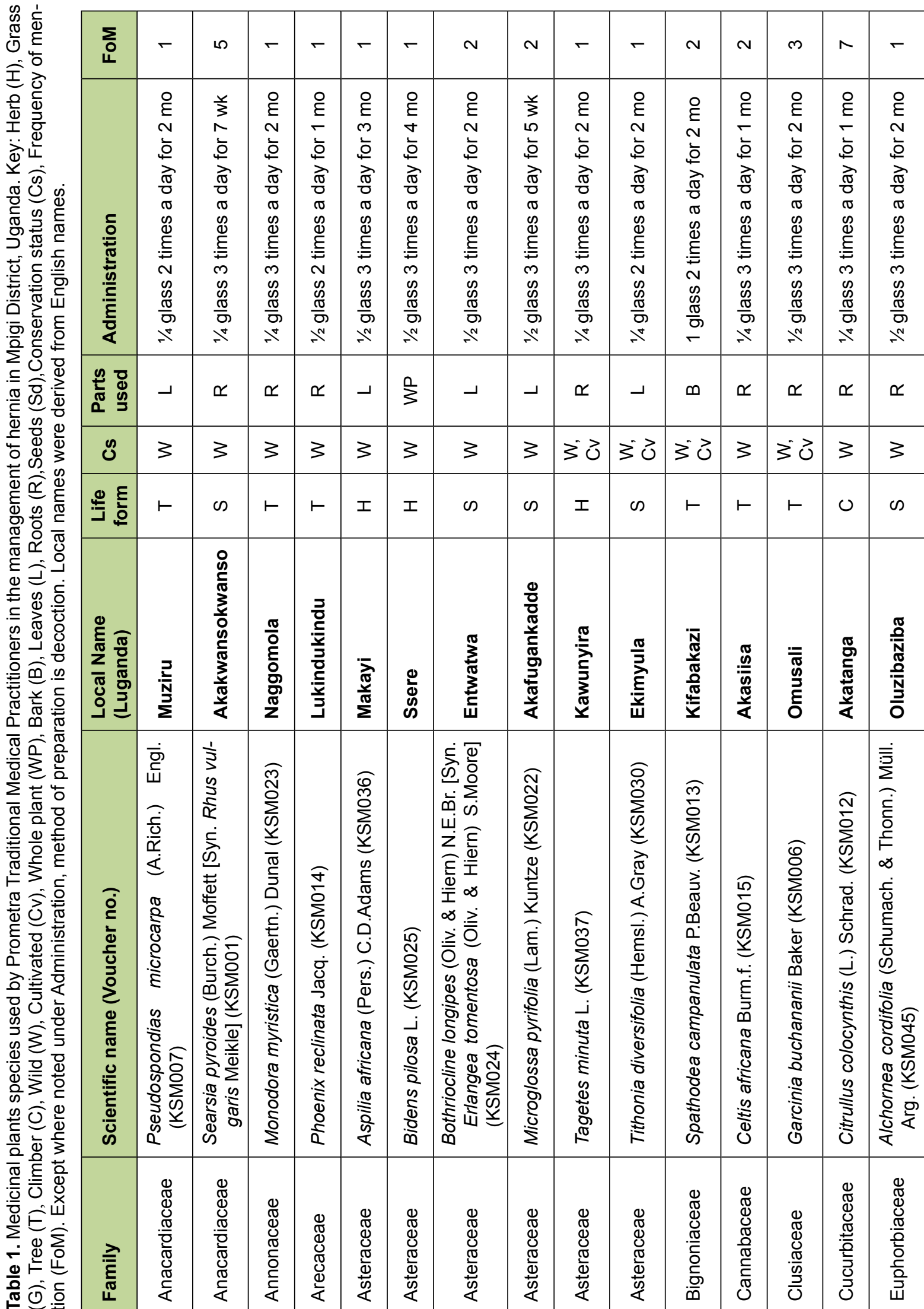


Kibuuka \& Anywar - Medicinal Plant Species Used in the Management of 293 Hernia by Traditional Medicine Practitioners in Central Uganda

\begin{tabular}{|c|c|c|c|c|c|c|c|c|c|c|c|c|c|c|c|c|c|}
\hline 인 & $N$ & 10 & $\infty$ & - & $r$ & $r$ & $r$ & $r$ & $\sim$ & $r$ & $m$ & $r$ & $m$ & $r$ & N & $\sim$ & $\nabla$ \\
\hline 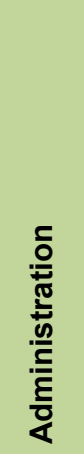 & 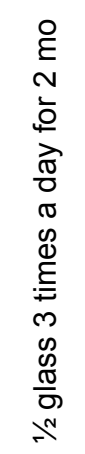 & 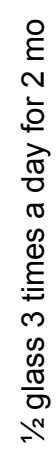 & 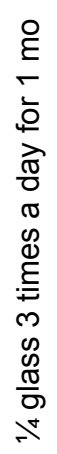 & 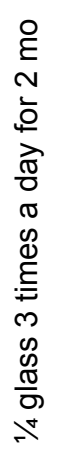 & 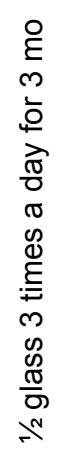 & 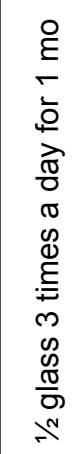 & 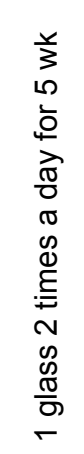 & 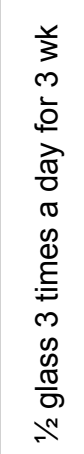 & 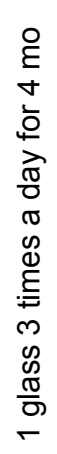 & 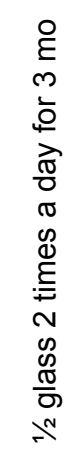 & 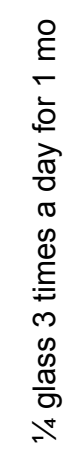 & 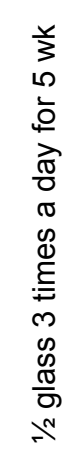 & 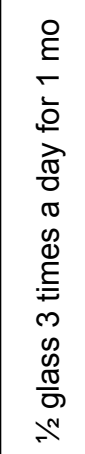 & 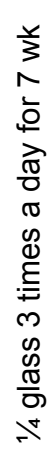 & 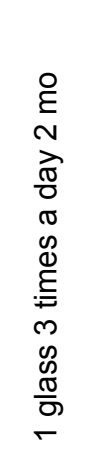 & 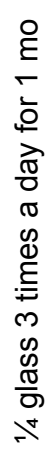 & 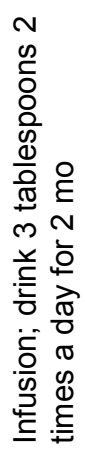 \\
\hline 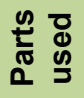 & $\varpi$ & \lrcorner & $\varpi$ & $\simeq$ & $\simeq$ & $\varpi$ & $\infty$ & $\varpi$ & $\varpi$ & $\begin{array}{l}\infty \\
- \\
-\end{array}$ & $\varpi$ & $\simeq$ & ـ & $\begin{array}{l}\text { D } \\
-\end{array}$ & $\frac{0}{3}$ & $\varpi$ & \lrcorner \\
\hline ơ & 30 & 3 & 3 & 3 & 3 & 30 & 30 & 3 & $\vec{U}$ & 3 & 3 & 3 & 30 & 3 & 30 & 3 & Uे \\
\hline 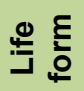 & $\vdash$ & $I$ & $\vdash$ & $\vdash$ & $\vdash$ & $\vdash$ & $\vdash$ & $\vdash$ & $I$ & $\omega$ & $\vdash$ & $\infty$ & $\infty$ & $I$ & $\infty$ & $\vdash$ & $\vdash$ \\
\hline 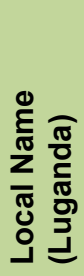 & $\begin{array}{l}\frac{\pi}{\pi} \\
\frac{\pi}{\pi} \\
\frac{\pi}{\pi} \\
\frac{\pi}{\sigma} \\
\frac{0}{\pi} \\
\Sigma\end{array}$ & 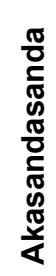 & 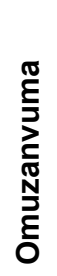 & 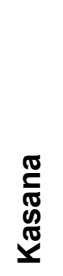 & 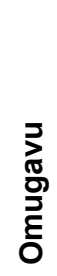 & 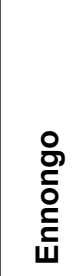 & 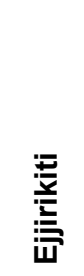 & $\begin{array}{l}\stackrel{0}{0} \\
\sum_{0}^{\circ} \\
\frac{0}{E} \\
\frac{0}{\omega}\end{array}$ & 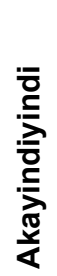 & 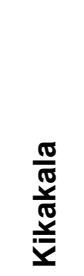 & 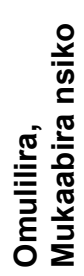 & 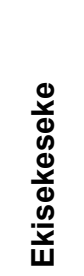 & 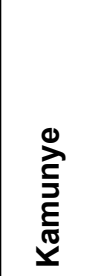 & 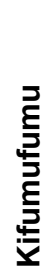 & 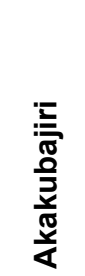 & 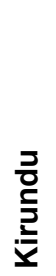 & 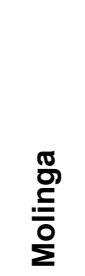 \\
\hline 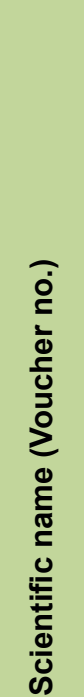 & 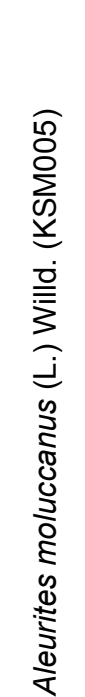 & 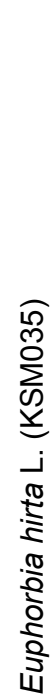 & 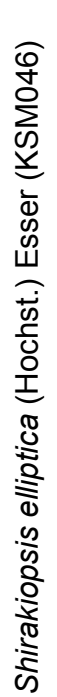 & 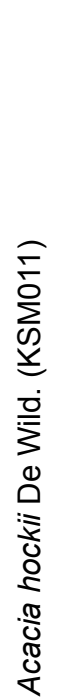 & 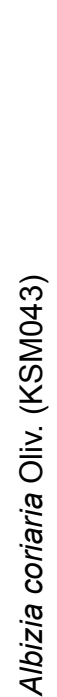 & 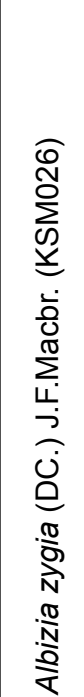 & 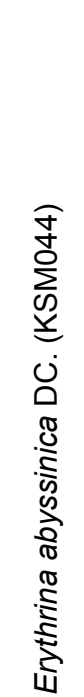 & 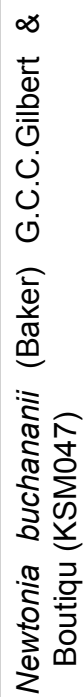 & 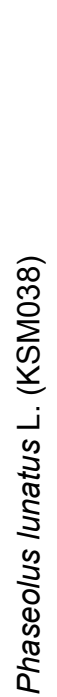 & 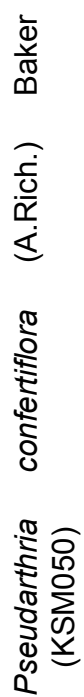 & 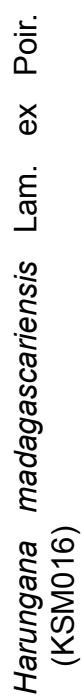 & 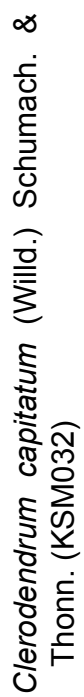 & 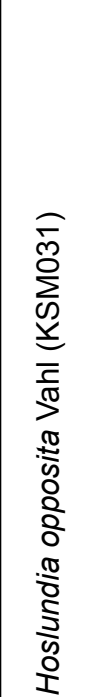 & 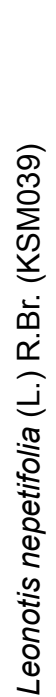 & 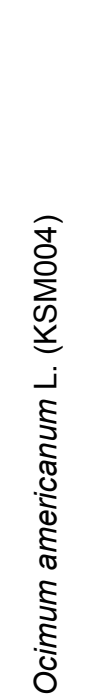 & 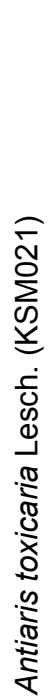 & 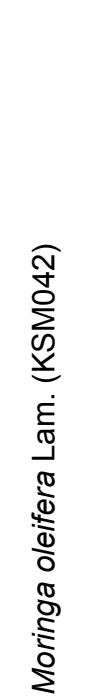 \\
\hline 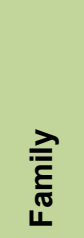 & 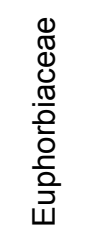 & 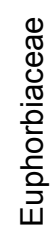 & 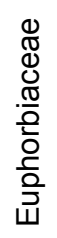 & $\begin{array}{l}\mathbb{\Xi} \\
\mathbb{\Xi} \\
\mathbb{\Xi} \\
\mathbb{\pi} \\
\mathbb{\varpi} \\
\leftarrow\end{array}$ & 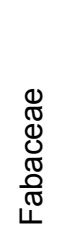 & $\begin{array}{l}\mathbb{0} \\
\mathbb{J} \\
\mathbb{J} \\
\mathbb{\pi} \\
\stackrel{0}{\pi} \\
\leftarrow\end{array}$ & 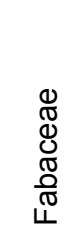 & 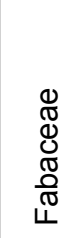 & $\begin{array}{l}\mathbb{\Xi} \\
\mathbb{\Xi} \\
\mathbb{U} \\
\mathbb{\pi} \\
\mathbb{0} \\
\leftarrow \\
\mathbb{1}\end{array}$ & 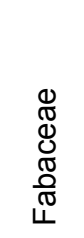 & 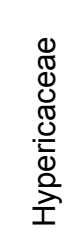 & 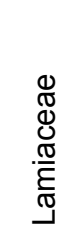 & 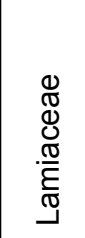 & 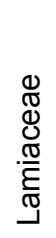 & 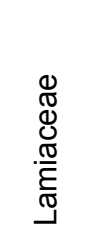 & 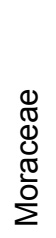 & 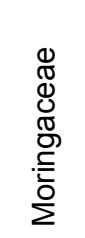 \\
\hline
\end{tabular}




\begin{tabular}{|c|c|c|c|c|c|c|c|c|c|c|c|c|c|c|c|c|c|c|c|}
\hline$\sum_{0}$ & m & $r$ & $N$ & - & $r$ & $\nabla$ & - & $\nabla$ & - & $N$ & $\sim$ & $r$ & $\sim$ & $N$ & $m$ & $\sim$ & $r$ & $\mp$ & $r$ \\
\hline 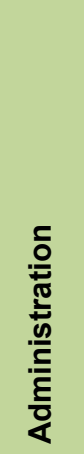 & 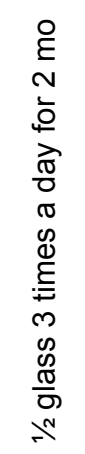 & 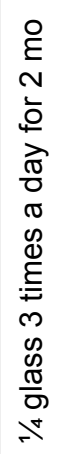 & 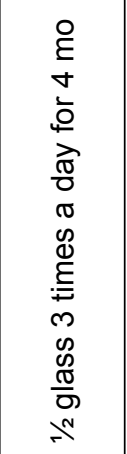 & 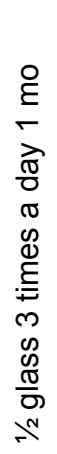 & 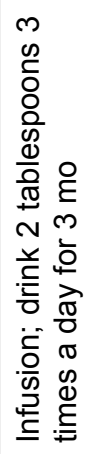 & 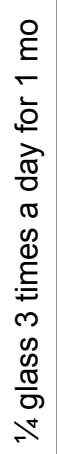 & 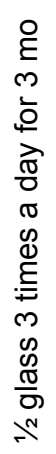 & 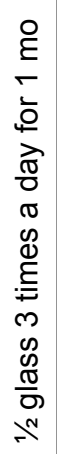 & 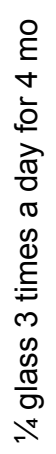 & 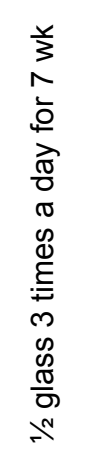 & 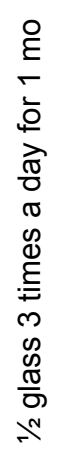 & 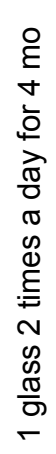 & 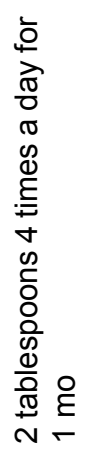 & 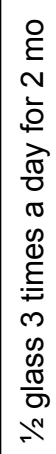 & 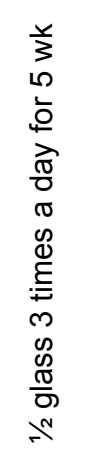 & 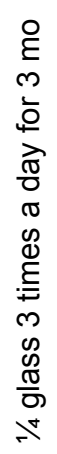 & 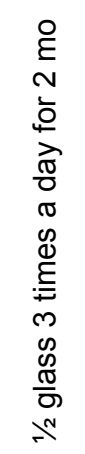 & 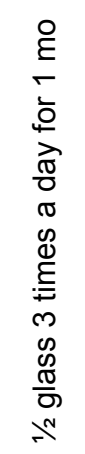 & 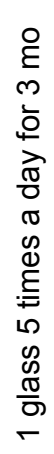 \\
\hline 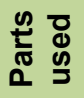 & $\simeq$ & 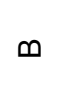 & 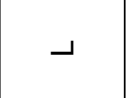 & $\varpi$ & 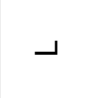 & $\propto$ & $\simeq$ & $\sum_{3}^{n}$ & $\varpi$ & 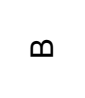 & $\varpi$ & $\simeq$ & க & \lrcorner & $\varpi$ & $\simeq$ & - & $\sum_{3}^{n}$ & $\simeq$ \\
\hline 0 & 30 & $\vec{U}$ & 3 & 3 & Uे & 3 & $\vec{U}$ & 3 & 3 & 30 & 3 & 3 & 3 & U & 3 & 3 & 30 & 3 & $\overrightarrow{0}$ \\
\hline$\stackrel{Ð}{\leftrightarrows}$ & $\vdash$ & $\vdash$ & I & $\vdash$ & (1) & (1) & 0 & $I$ & $\vdash$ & $\vdash$ & $\vdash$ & $\omega$ & $\vdash$ & $\vdash$ & $\vdash$ & $I$ & $\infty$ & 0 & $I$ \\
\hline 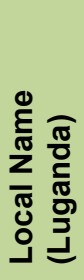 & 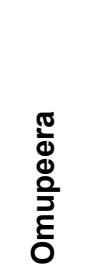 & $\begin{array}{l}\frac{\pi}{5} \\
\frac{0}{\varepsilon} \\
\frac{\pi}{J}\end{array}$ & 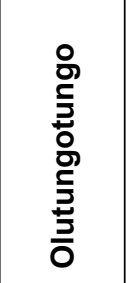 & 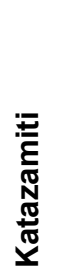 & $\begin{array}{l}\overline{0} \\
\frac{0}{\bar{n}} \\
\underline{\underline{x}}\end{array}$ & 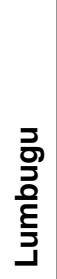 & 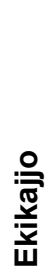 & $\begin{array}{l}\frac{\pi}{0} \\
\frac{1}{\pi} \\
3 \\
0\end{array}$ & $\begin{array}{l}0 \\
\frac{0}{0} \\
0 \\
\frac{3}{0} \\
\frac{0}{0} \\
\frac{0}{0} \\
\frac{3}{y}\end{array}$ & $\begin{array}{l}\frac{\bar{N}}{D} \\
\stackrel{D}{E} \\
0\end{array}$ & 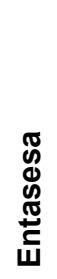 & 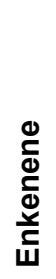 & 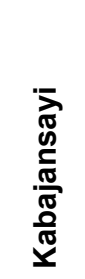 & . & 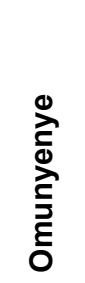 & 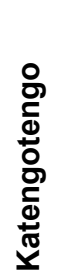 & 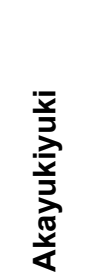 & 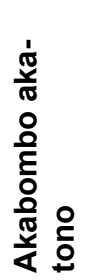 & 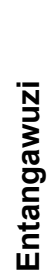 \\
\hline 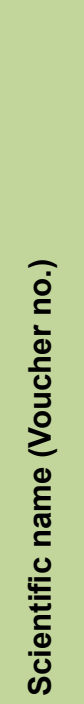 & 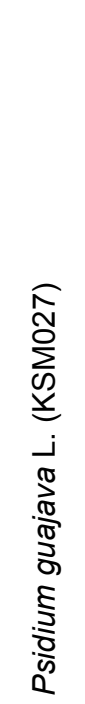 & 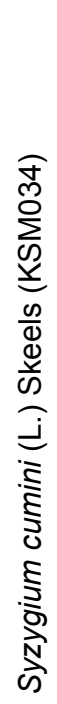 & 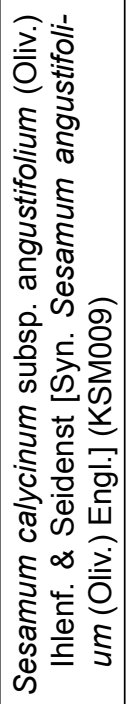 & 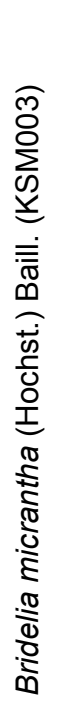 & 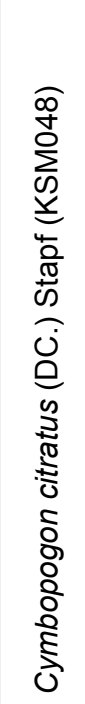 & 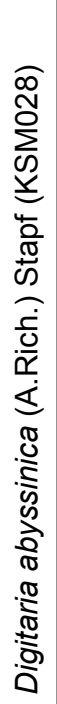 & 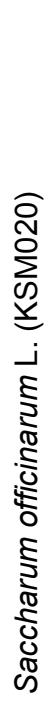 & 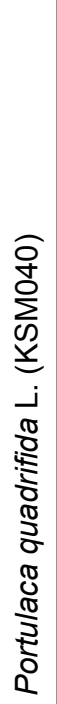 & 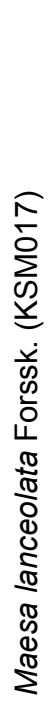 & 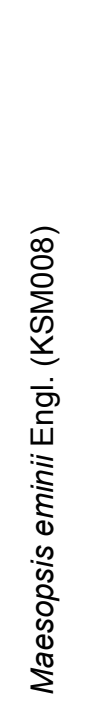 & 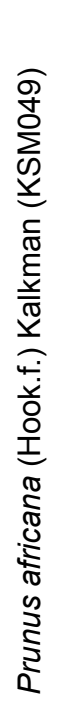 & 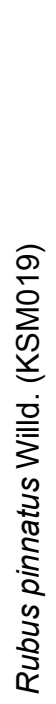 & 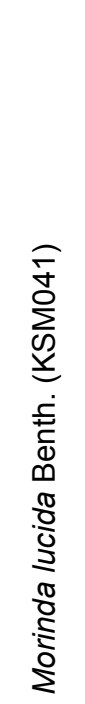 & 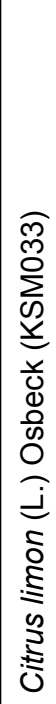 & 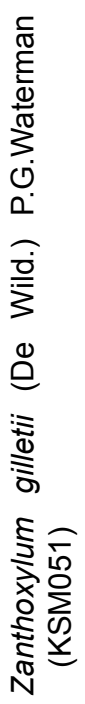 & 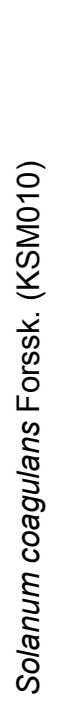 & 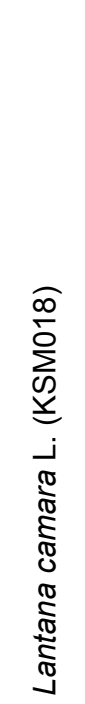 & 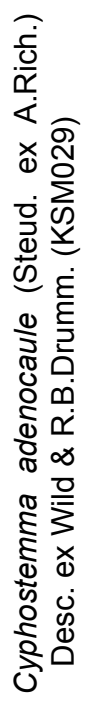 & 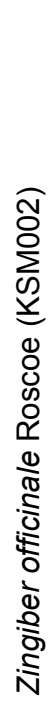 \\
\hline 离 & 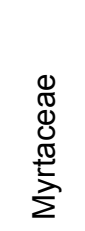 & 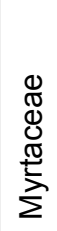 & 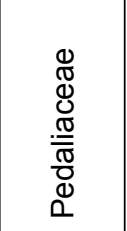 & 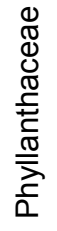 & 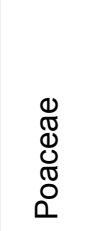 & $\begin{array}{l}\mathbb{N} \\
\mathbb{J} \\
\mathbb{N} \\
\mathbb{\sigma}\end{array}$ & $\begin{array}{l}\mathbb{1} \\
\mathbb{\Xi} \\
\mathbb{J} \\
\mathbb{\sigma}\end{array}$ & 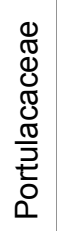 & 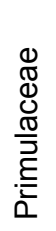 & 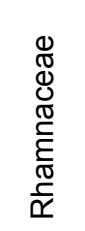 & 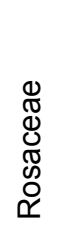 & 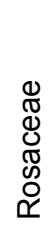 & $\begin{array}{l}\mathbb{0} \\
\mathbb{0} \\
0 \\
\frac{0}{0} \\
\frac{0}{0} \\
\frac{0}{2} \\
\frac{\alpha}{1}\end{array}$ & $\begin{array}{l}\mathbb{\pi} \\
\mathbb{d} \\
0 \\
\mathbb{J}\end{array}$ & 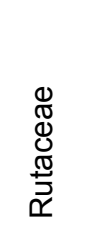 & 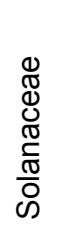 & 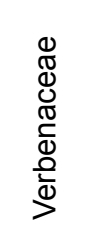 & $\begin{array}{l}\mathbb{J} \\
\mathbb{0} \\
\mathbb{0} \\
\stackrel{\mathbb{\pi}}{*}\end{array}$ & 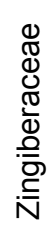 \\
\hline
\end{tabular}




\section{Kibuuka \& Anywar - Medicinal Plant Species Used in the Management of Hernia by Traditional Medicine Practitioners in Central Uganda}

quently used method of preparation (96\%), and infusions $(4 \%)$ were the least frequently used. Most of the medicines were administered to patients orally.

\section{Discussion}

\section{Traditional concept of hernia}

From this study, it becomes apparent that the TMPs have a traditional concept of hernia. It includes the etiology of hernia and treatment using non-surgical interventions particularly herbal medicines. The TMPs also advocate for diet modification by discouraging the frequent over-consumption of starchy foods, which they believe contribute to the weakening of the abdominal walls. They argue that since a starch-laden diet can cause constipation, a similar effect may be experienced within the intestinal walls, thus causing hernia.

\section{Medicinal plants used to manage hernia}

The traditional healers use various medicinal plant species for treating hernia. It is also important to note that many of plant species mentioned were also used to treat other diseases. However, unlike this study whose focus was on medicinal plants used in the treatment of hernia, other studies have casually documented some medicinal plant species used in hernia treatment.

Only Hoslundia opposita Vahl, Bridelia micrantha (Hochst.) Baill., and Erythrina abyssinica DC. from this study have also been reported in other studies as being used in the treatment of hernias (Amri \& Kisangau 2012, Gradé et al. 2009, Namukobe et al. 2011, Tabuti et al. 2003). The rest of the plant species have not been recorded prior to this study as being used in the treatment of hernia.

The diagnosis of hernia by traditional healers is similar to the method of physical examination used in western medicine. Odula and Kakande (2004), recommended meticulous physical examination and early diagnosis as some of the means of reducing the morbidity associated with hernia surgery.

\section{Possible mechanism of action of some of the plants used to treat hernia}

Claims over therapeutic values of medicinal plants require evaluation to determine their efficacy and assess potential toxic effects. This process of validation builds confidence among users of these medicines. This in turn encourages greater reliance on traditional medicine in primary health care (WHO 1987). Patients with inguinal hernia have been shown to have a defective collagen synthesis mechanism coupled with changes in the ratios of the types of collagen. This renders the abdominal sinews weaker, making them more prone to hernias (Ajabnoor et al. 1992, Rosch et al. 2002, Tharappel 2014, Wagh \& Read 1972).

The ability of some plant species to stimulate the synthesis of collagen has been demonstrated. Studies have shown the plant extracts of Eucommia ulmoides and Acalypha indica to promote collagen synthesis in vitro (Ganeshkumar et al. 2012, Li et al. 2000). This phenomenon could explain the healing claimed by the TMPs, since some of the medicinal plants documented in this study could be acting via the same mechanism. It is therefore critical that scientific investigations into the efficacy and possible mode of action of such medicinal plants are carried out.

\section{Harvesting, preparation, and administration}

During the survey, it was noted that the frequency of harvest and use of any medicinal plant depended on the availability of alternative plant species for treating the patient. In addition, the number of patients to be treated determined the frequency of harvest of a particular medicinal plant. Decoctions were prepared by boiling the plant material in water for up to two or three hours. Infusions on the other hand were prepared by soaking the plant material in hot water for up to 30 minutes.

Most of the plants were used in mixtures, especially the woody or hard parts of plants. According to traditional healers, plants when used in mixtures are more effective due to their combined effects than when used singly. Similar results were reported by Kakudidi et al. (2000) in and around Kibale National Park. Traditional healers recommended high levels of hygiene during the preparation, handling, and administration of the medicines because they were administered orally. Okello and Ssegawa (2007) made similar observations among traditional healers in northern Uganda. It was also noted that some medicinal plant species were referred to by more than one local name (e.g., Harungana madagascariensis Lam. ex Poir.). This served the purpose of concealing their identities from competitors. Similar trends have been reported in South Africa and other parts of Uganda for the purpose of hiding knowledge from outsiders (Cunningham 1993, Okello \& Ssegawa 2007).

\section{Conservation issues}

Although most of the plants species documented were harvested from the wild, some of them were not readily available and were therefore cultivated in home gardens (e.g., Zingiber officinale Roscoe, Saccharum officinarum L., Cymbopogon citratus (DC.) Stapf, Phaseolus lunatus, and Moringa oleifera Lam.). Some of the plant species used to manage hernia were threatened by indiscriminate and overharvesting, particularly the roots and bark. For example Newtonia buchananii (Baker) G.C.C.Gilbert \& Boutiqu, Prunus africana (Hook.f.) Kalkman, and Aleurites moluccanus (L.) Willd. were found to be heavily debarked. 
Since the roots and bark were the most frequently used parts in the preparation of herbal remedies, such poor harvesting techniques affect the ability of the plants to regenerate and survive. This is particularly true for slowgrowing woody species. The increased rate of land conversion for agriculture has also led to loss of natural habitats and made it difficult to obtain certain plant species in the wild (e.g., Microglossa pyrifolia (Lam.) Kuntze). Tabuti et al. (2003) and Anywar et al. (2014) reported similar findings in other parts of Uganda. Therefore, there is a need to initiate both in situ and ex situ conservation of programs at different community levels as well as sensitization of the traditional healers.

\section{Knowledge dynamics of medicinal plants}

The knowledge about these medicinal plants was acquired and passed on orally from the elders to the young people. Some of the knowledge was gained through trainings from organizations such as Prometra. Most of the TMPs involved in hernia treatment were female. Attempts to pass on this knowledge to their children were hampered by lack of interest and the fact that the children spend most of their time in school. Similar reasons were advanced in a study conducted by Anywar et al. (2014) in northwestern Uganda.

\section{Conclusion}

TMPs from Prometra Uganda have a traditional concept of hernia linked to the regular overconsumption of monotonous starchy diets. This eventually weakens the intestinal walls. In the long run, this causes the internal organs to protrude through the weakened skin causing hernia. Hernia is diagnosed by physically examining the patient. Even though hernia is managed surgically in western medicine, the TMPs use several locally available medicinal plant species to treat the disease. The treatment often lasts between 1-4 months, with TMPs claiming complete healing.

However, some of the medicinal plants are threatened by the high rates of land conversion and poor, unsustainable harvesting methods. Lack of interest by the younger generation in learning about these herbs is also a threat to this hitherto undocumented indigenous knowledge.

\section{Future research}

These plant species and practices by the traditional medicine practitioners need to be investigated further to validate claims of healing. The investigations should involve ascertaining the safety, efficacy, chemical constituents, and possible mode of action of these plant species.

\section{Acknowledgments}

We would like to thank the TMPs of Prometra Uganda for participating in this study. We also want to thank Dr. Sekajja Yahya of Prometra for granting us access to healers, and Dr. Alex Upoki at the Department of Surgery, Mulago National referral hospital and Makerere University College of Health Sciences, for invaluable advice and guidance during the writing of the manuscript.

\section{Literature Cited}

Ajabnoor, M.A., A.M. Mokhtar, A.A. Rafee \& A.M. Taha. 1992. Defective collagen metabolism in Saudi patients with hernia. Annals of Clinical Biochemistry 29(4):430436. http://dx.doi.org/10.1177/000456329202900411

Amri, E. \& D.P. Kisangau. 2012. Ethnomedicinal study of plants used in villages around Kimboza forest reserve in Morogoro, Tanzania. Journal of Ethnobiology and Ethnomedicine 8:1. http://dx.doi.org/10.1186/1746-4269-8-1

Anywar, G., H. Oryem-Origa \& M.K. Mugisha. 2014. Wild plants used as nutraceuticals from Nebbi District, Uganda. European Journal of Medicinal Plants 4(6):641-660. http://dx.doi.org/10.9734/EJMP/2014/7634

Beddy, P., P.F. Ridgway, T. Geoghegan, C. Peirce, P. Govender, F.B.V. Keane, W.C. Torreggiani \& K.C.P. Conlon. 2006. Inguinal hernia repair protects testicular function: $A$ prospective study of open and laparoscopic herniorraphy. Journal of the American College of Surgeons 203(1):1723. http://dx.doi.org/10.1016/j.jamcollsurg.2006.04.013

Burcharth, J., M. Pedersen, T. Bisgaard, C. Pedersen \& J. Rosenberg. 2013. Nationwide prevalence of groin hernia repair. PLOS ONE 8(1):e54367. http://dx.doi.org/10.1371/ journal.pone.0054367

Cunningham, A.B. 1993. African Medicinal Plants: Setting priorities at the interface between conservation and primary health care. People and Plants working paper 1. United Nations Educational, Scientific and Cultural Organization (UNESCO), Paris, France. http://unesdoc.unesco.org/images/0009/000967/096707E.pdf

Dabbas, N., K. Adams, K. Pearson \& G.T. Royle. 2011. Frequency of abdominal wall hernias: Is classical teaching out of date? Journal of the Royal Society of Medicine Short Reports 2(1):5. http://dx.doi.org/10.1258/ shorts.2010.010071

Everhart, J.E. 1994. Abdominal wall hernia. Pp. 469-507 in Digestive Diseases in the United States: Epidemiology and impact. Edited by J.E. Everhart. U.S. Department of Health and Human Services, Public Health Service, Na- 


\section{Kibuuka \& Anywar - Medicinal Plant Species Used in the Management of Hernia by Traditional Medicine Practitioners in Central Uganda}

tional Institutes of Health No. 94-1447. Washington, D.C., U.S.A.

Ganeshkumar, M., T. Ponrasu, R. Krithika, K. Iyappan, V.S. Gayathri \& L. Suguna. 2012. Topical application of Acalypha indica accelerates rat cutaneous wound healing by up-regulating the expression of Type I and III collagen. Journal of Ethnopharmacology 142(1):14-22. http:// dx.doi.org/10.1016/j.jep.2012.04.005

Gradé, J., R. Weladji, J. Tabuti \& P. Van Damme. 2009. Healer-driven ethnoveterinary knowledge diffusion among semi-nomadic pastoralists in Karamoja, Uganda. Afrika Focus 22(1):57-75.

Heckathorn, D.D. 2011. Comment: Snowball versus respondent-driven sampling. Sociology Methodology 41(1):355-366. http://dx.doi.org/10.1111/j.1467$\underline{9531.2011 .01244 . x}$

Kakudidi, E.K.Z., R. Bukenya-Ziraba \& J.M. Kasenene. 2000. The medicinal plants in and around Kibale National Park in Western Uganda. Norwegian Journal of Botany (Lidia) 5(4):109-124.

Kingsworth, A. \& K. LeBlanc. 2003. Hernias: Inguinal and incisional. The Lancet 362(9395):1561-1571. http:// dx.doi.org/10.1016/S0140-6736(03)14746-0

Li, Y., S. Kamo, K. Metori, K. Koike, Q.-M. Che \& S. Takahashi. 2000. The promoting effect of Eucommiol from Eucommiae cortex on collagen synthesis. Biological and Pharmaceutical Bulletin 23(1):54-59. http://dx.doi. org/10.1248/bpb.23.54

Martin, G.J. 1995. Ethnobotany: A methods manual. Chapman \& Hall, London, U.K.

Namukobe, J., J.M. Kasenene, B.T. Kiremire, R. Byamukama, M. Kamatenesi-Mugisha, S. Krief, V. Dumontet \& J.D. Kabasa. 2011. Traditional plants used for medicinal purposes by local communities around the Northern sector of Kibale National Park, Uganda. Journal of Ethnopharmacolog 136(1):236-245. http://dx.doi.org/10.1016/j. jep.2011.04.044

Njoroge, G.N. \& J.W. Kibunga. 2007. Herbal medicine acceptance, sources and utilization for diarrhoea management in a cosmopolitan urban area (Thika, Kenya). African Journal of Ecology 45(S1):65-70. http://dx.doi. org/10.1111/j.1365-2028.2007.00740.x

NPHC (National Population and Housing Census). 2014. Provisional Results for Uganda Census (2014). Revised Edition. Uganda Bureau of Statistics, Kampala, Uganda.

Odula, P.O. 2011. Busoga hernia prevalence in abdominal emergencies. Africa Journal of Surgery 9(1):76-54.
Odula, P.O. \& I. Kakande. 2004. Groin hernia in Mulago Hospital, Kampala. East and Central Africa Journal of Surgery 9(1):48-52.

Okello, J. \& P. Ssegawa. 2007. Medicinal plants used by communities of Ngai Subcounty, Apac District, northern Uganda. African Journal of Ecology 45(S1):76-83. http:// dx.doi.org/10.1111/j.1365-2028.2007.00742.x

Rosch, R., U. Klinge, Z. Si, K. Junge, B. Klosterhalfen \& V. Schumpelick. 2002. A role for the collagen I/III and MMP1/-13 genes in primary inguinal hernia? BMC Medical Genetics 3:2. http://dx.doi.org/10.1186/1471-2350-3-2

Ross, I.A. 2003. Medicinal Plants of the World: Chemical constituents, traditional and modern medicinal uses. Volume 1, second edition. Humana Press Inc., Totowa, New Jersey, U.S.A. http://dx.doi.org/10.1007/978-1-59259$\underline{365-1}$

Ruhl, C.E. \& J.E. Everhart. 2007. Risk factors for inguinal hernia among adults in the US population. American Journal of Epidemiology 165(10):1154-1161. http://dx.doi. org/10.1093/aje/kwm011

Tabuti, J.R.S., C.B. Kukunda \& P.J. Waako. 2010. Medicinal plants used by traditional medicine practitioners in the treatment of tuberculosis and related ailments in Uganda. Journal of Ethnopharmacology 127(1):130-136. http:// dx.doi.org/10.1016/j.jep.2009.09.035

Tabuti, J.R.S., K.A. Lye \& S.S. Dhillion. 2003. Traditional herbal drugs of Bulamogi, Uganda: Plants, use and administration. Journal of Ethnopharmacology 88(1):19-44. http://dx.doi.org/10.1016/S0378-8741(03)00161-2

Tekatli, H., N. Schouten, T. van Dalen, I. Burgmans \& N. Smakman. 2012. Mechanism, assessment, and incidence of male infertility after inguinal hernia surgery: A review of the preclinical and clinical literature. The American Journal of Surgery 204(4):503-509. http://dx.doi.org/10.1016/j. amjsurg.2012.03.002

Tharappel, J.C., C.E. Bower, J.W. Harris, S.K. Ramineni, D.A. Puleo \& J.S Roth. 2014. Doxycycline administration improves fascial interface in hernia repair. Journal of Surgical Research 190(2):692-698. http://dx.doi. org/10.1016/j.jss.2014.05.013

UDIH (Uganda Districts Information Handbook). 2012. Uganda Districts Information Handbook. 2011-2012 expanded edition. Fountain Publishers, Kampala, Uganda.

Wagh, P.V. \& R.C. Read. 1972. Defective collagen synthesis in inguinal herniation. The American Journal of Surgery 124(6):819-822. http://dx.doi.org/10.1016/00029610(72)90148-1 
WHO (World Health Organization). 1987. The Promotion and Development of Traditional Medicine. Technical Report Series, No. 622, World Health Organization, Geneva, Switzerland. http://apps.who.int/iris/bitstream/10665/40995/1/WHO TRS 622.pdf
Zollinger, R.M., Jr. 2003. Classification systems for groin hernias. Surgical Clinics of North America 83(5):10531063. http://dx.doi.org/10.1016/s0039-6109(03)00126-9 